\title{
The Neural Basis of Temporal Order Processing in Past and Future Thought
}

\author{
Arnaud D'Argembeau, Olivier Jeunehomme, Steve Majerus, \\ Christine Bastin, and Eric Salmon
}

\begin{abstract}
Although growing evidence has shown that remembering the past and imagining the future recruit a common core network of frontal-parietal-temporal regions, the extent to which these regions contribute to the temporal dimension of autobiographical thought remains unclear. In this fMRI study, we focused on the event-sequencing aspect of time and examined whether ordering past and future events involve common neural substrates. Participants had to determine which of two past (or future) events occurred (or would occur) before the other, and these order judgments were compared with a task requiring to think about the content of the same past or future events. For both past and future events, we found that the left posterior hippocampus was more activated when establishing the order of events, whereas the anterior hippocampus was more acti-
\end{abstract}

vated when representing their content. Aside from the hippocampus, most of the brain regions that were activated when thinking about temporal order (notably the intraparietal sulcus, dorsolateral pFC, dorsal anterior cingulate, and visual cortex) lied outside the core network and may reflect the involvement of controlled processes and visuospatial imagery to locate events in time. Collectively, these findings suggest (a) that the same processing operations are engaged for ordering past events and planned future events in time, (b) that anterior and posterior portions of the hippocampus are involved in processing different aspects of autobiographical thought, and (c) that temporal order is not necessarily an intrinsic property of memory or future thought but instead requires additional, controlled processes.

\section{INTRODUCTION}

Humans live in subjective time (Suddendorf \& Corballis, 2007; Tulving, 2002): We have internalized a view of the past and future as parts of a temporal framework filled with the happenings of our life (Friedman, 2005), and much of our everyday thinking consists in mental travels to such past and future times (D'Argembeau, Renaud, \& Van der Linden, 2011; Berntsen \& Jacobsen, 2008; Klinger \& Cox, 1987). This ability to conceive of nonpresent times has recently attracted growing attention in psychology and neuroscience because of its key role in many fundamental aspects of human cognition and behavior, such as planning, decision-making, and self-regulation (Schacter, 2012; Szpunar, 2010; Boyer, 2008; Suddendorf \& Corballis, 2007; Atance \& O'Neill, 2001).

Although considerable progress has been made in elucidating how the content of past and future thoughts is constructed and elaborated (for review, see Mullally \& Maguire, 2014; D’Argembeau, 2012; Schacter et al., 2012; Szpunar, 2010), the processing of time itself remains elusive, especially as regards to prospective thought (Klein, 2013; Szpunar, 2011; Friedman, 2005). Various kinds of temporal information can potentially be available when

University of Liège

(C) 2014 Massachusetts Institute of Technology

we think about past and future events (e.g., dates vs. feelings of distance; Skowronski \& Sedikides, 2007; Friedman, 1993, 2005). Among these, the event-sequencing aspect of time (i.e., the way events are temporally ordered with respect to each other) may be particularly important for keeping track of goal progress and for planning and organizing behavior (Conway, 2009). Previous research has shown that people have some knowledge about the order of past events (St Jacques, Rubin, LaBar, \& Cabeza, 2008; Skowronski, Walker, \& Betz, 2003), but there is less evidence that order information is coherently represented for envisioned future events (Friedman, 2005). Furthermore, it is unknown whether similar or distinct neurocognitive processes are involved in ordering past and future events in time.

Here, we sought to address these questions by identifying possible commonalities and differences in the neural basis of temporal order processing in past and future thought. Important insights into the neurocognitive underpinnings of prospective thought have recently been gained from functional neuroimaging. Indeed, a growing number of studies have shown that remembering past events and imagining future events recruit a common "core" network of frontal, parietal, and temporal regions (for a review, see Schacter et al., 2012), suggesting that past and future thoughts involve common representations and processes 
(Buckner \& Carroll, 2007; Hassabis \& Maguire, 2007; Schacter \& Addis, 2007; Suddendorf \& Corballis, 2007). Whether such commonalities embrace temporal information is unclear, however. On the one hand, it has been found that several regions of the core network (notably the hippocampus) are activated when imagining events or scenes that are not located at particular times in the past or future (Summerfield, Hassabis, \& Maguire, 2010; Hassabis, Kumaran, \& Maguire, 2007), suggesting that these regions support nontemporal processes (Mullally \& Maguire, 2014; Eacott \& Easton, 2012; Hassabis \& Maguire, 2007). On the other hand, there is evidence that the hippocampus is activated when remembering temporal order (e.g., Ekstrom, Copara, Isham, Wang, \& Yonelinas, 2011; Lehn et al., 2009), suggesting that the hippocampus plays a role in the temporal/ sequential organization of memory (Eichenbaum, 2013).

Besides the hippocampus, other candidate regions for processing temporal order in past and future thought include lateral prefrontal and parietal cortices. The $\mathrm{pFC}$ has long been hypothesized to play a key role in the processing of time (Wheeler, Stuss, \& Tulving, 1997; Ingvar, 1985) - in particular, the temporal organization of behavioral sequences (Fuster, 2001)—and neuroimaging studies have demonstrated activations of lateral prefrontal regions when people remember the order of past events (e.g., Ekstrom et al., 2011; St Jacques et al., 2008). Moreover, temporal information is often construed in terms of spatial representations (e.g., mapping events along a mental time line; Nunez \& Cooperrider, 2013; Christian, Miles, \& Macrae, 2012; Arzy, Adi-Japha, \& Blanke, 2009; Boroditsky, 2000), and the parietal cortex may play a role in this process (Bueti \& Walsh, 2009). Two recent studies indeed found activations in prefrontal and parietal regions when processing temporal information in future thought (Nyberg, Kim, Habib, Levine, \& Tulving, 2010; Arzy, Collette, Ionta, Fornari, \& Blanke, 2009), but the exact functions of these regions remain unclear and the extent to which they contribute to temporal order processing is unknown.

Functional neuroimaging provides an interesting tool for investigating whether similar or distinct processing operations are involved in establishing temporal order in past and future thought (Mather, Cacioppo, \& Kanwisher, 2013). One possibility is that order is processed differently for retrospective and prospective thought because of an inherent asymmetry between the two sides of time (Suddendorf, 2010): Past events already occurred and cannot be changed, so order is fixed and determined; future events have yet to happen and cannot be known with certainty, so order is more malleable. If this asymmetry is mirrored in the mechanisms used to process temporal order, distinct (or at least partly distinct) brain regions should be implicated in determining the order of past and future events. On the other hand, research suggests that we rarely have direct access to temporal information and determining the times of past events often involves reconstructive processes, such as inferring the locations of events within conventional time patterns (e.g., parts of the day, week, or year; Friedman,
1993, 2004). Similar location-based processes could be involved in determining the times of future events (Friedman, 2005), in which case largely overlapping neural activations should be observed when ordering past and future events (quantitative differences could still be apparent, however, for example, if order processing is more difficult for one kind of events than for the other).

To test these predictions, participants were scanned while completing a temporal judgment task that required them to determine which of two past events occurred before the other (past order condition) or which of two planned future events would occur before the other (future order condition); the events were selected from individualized prescan interviews in which participants were asked to recall a series of events that happened to them in the past week and to imagine a series of events that they think will happen to them in the next week. This order judgment task was compared with a task requiring to think about the content of the same past and future events: Participants were presented with pairs of events containing a past or future event they generated during the prescan interview and an event that did not happen to them in the past week or would not happen to them in the next week, and they had to determine which of the two events belongs to their personal past or future (past and future event conditions). The four conditions thus constituted a 2 (task: order vs. event) $\times 2$ (temporal orientation: past vs. future) factorial design that allowed us to investigate commonalities and differences in the neural processing of temporal order in past and future thought.

To further investigate whether similar processes are involved in determining the order of past and future events, we also varied the temporal distance between the two events presented in the order judgment task. Previous research has evidenced a temporal distance effect in order judgments for past events: The farther apart in time the two events are, the easier the judgment becomes (e.g., RT decreases; Skowronski et al., 2003). If similar processing operations are involved in determining the order of past and future events, increasing the difficulty of order judgments (by decreasing temporal distance between events) should have similar effects for both types of events in terms of behavioral performance and neural activity.

\section{METHODS \\ Participants}

Participants were 22 right-handed young adults ( 13 women; mean age $=21$ years, range $=18-25$ years) who reported no history of psychiatric or neurological disorders and no current use of any psychoactive medications. Two additional participants were run but excluded from data analysis because of task noncompliance or excessive head movement during image acquisition. All participants gave their written informed consent to take part in the study, which 
was approved by the ethics committee of the Medical School of the University of Liège.

\section{Materials and Procedures}

\section{Prescan Interview}

The day before the fMRI session, participants were asked to recall a series of events that happened to them in the past week and to imagine a series of events that they thought would happen to them in the next week. The instructions specified that the recalled and imagined events should be specific (unique events that happen at a particular place and time) and not routine events that could happen on any day; examples were provided to illustrate what would or would not be considered as specific events. The experimenter then went through the past 7 days and the next 7 days one by one with the participant to collect 10 specific events for each temporal orientation (i.e., past and future; for the future period, only events that would happen after the scanning session planned on the next day were considered). For each reported event, the participant indicated the day on which this event happened (or would likely happen) and provided a short sentence summarizing the essence of the event; these sentences included information about a place, person, and/or activity that individualized each reported event but did not include temporal information (e.g., "to fetch my parents at the airport").

\section{fMRI Session}

Participants performed four tasks while in the scanner. Two tasks consisted of determining the temporal order of event occurrence, either in the past (past order condition) or in the future (future order condition). In both tasks, participants were presented with pairs of past or future events and had to judge which of the two events occurred before the other (in the past order condition) or would occur before the other (in the future order condition). Each of the 10 past events and 10 future events generated in the prescan interview were presented three times, in association with different events, such that a total of 30 pairs of events were presented for each temporal orientation. The lag between the two events of each pair ranged between 1 and 6 days, and care was taken to match the past and future conditions in this regard; the mean average lag was 2.77 days in the past condition and 2.33 days in the future condition, $t(21)=0.49, p=.63$, and the mean standard deviation of the lag was 1.54 in the past condition and 1.48 in the future condition, $t(21)=$ $0.96, p=.35$.

In the two other tasks, participants were presented with pairs of events that included one of the past or future events that had been generated during the prescan interview and one event that did not happen to them in the past week or would not likely happen to them in the next week (i.e., an event taken from another participant). For each pair, participants had to select which of the two events belonged to their personal past (past event condition) or future (future event condition). Each of the 10 past events and 10 future events generated in the prescan interview were presented three times, in association with different nonpersonal events (in total, there were 10 nonpersonal past events and 10 nonpersonal future events that were each presented three times), such that a total of 30 pairs of events were presented for each temporal orientation.

The 30 trials of each task were presented in blocks of three trials. Each block started with a cue slide (2 sec) indicating which task participants had to perform (e.g., future order). Then, three pairs of events were presented successively for 6 sec each. For each pair, the two sentences summarizing the events were presented above each other and were separated by the symbol "-." Participants pressed one of two designated buttons on a response box to select which of the two events happened or would happen before the other (in the order conditions) or to select which of the two events belonged to their personal past or future (in the event conditions). They were further instructed to keep thinking about the time when the events occurred or would occur (in the order conditions) or about the content of the personal events (in the event conditions) during the entire duration of presentation of the pairs on the screen (i.e., during the $6 \mathrm{sec}$ ). All trials were separated by a fixation cross of variable duration (lasting between 2 and $3 \mathrm{sec}$ ). Ten blocks of trials for each condition were presented in a pseudorandom order such that a particular condition could not be repeated immediately and could not be separated by more than six blocks of a different condition. Within blocks of the order conditions, the order of presentation of the different time lags between the two events of each pair was determined randomly. Immediately before scanning, participants were presented with some practice trials (using fictive events that did not belong to their personal past or future) to familiarize them with the tasks.

\section{Postscan Interview}

Postscan interviews were conducted to assess the extent to which participants followed the instructions during the scanning session. All participants indicated that they made order judgments by thinking about the time when the events occurred (for past events) or would occur (for future events) rather than attempting to remember the dates they provided during the prescan interview. One participant mentioned that the expected date of a future event had changed since the prescan interview and that he made order judgments in reference to this new schedule during scanning; therefore, this new expected date of the future event was taken into account for computing correct responses. 


\section{fMRI Data Acquisition}

fMRI time series were acquired on a 3T head-only scanner (Magnetom Allegra, Siemens Medical Solutions, Erlangen, Germany) operated with the standard transmit-receive quadrature head coil. Multislice $\mathrm{T}_{2} *$-weighted functional images were acquired with a gradient-echo EPI sequence using axial slice orientation and covering the whole brain (34 slices, field of view $[\mathrm{FoV}]=192 \times 192 \mathrm{~mm}^{2}$, voxel size $3 \times 3 \times 3 \mathrm{~mm}^{3}, 25 \%$ interslice gap, matrix size $64 \times 64 \times$ 34 , repetition time $[\mathrm{TR}]=2040 \mathrm{msec}$, echo time $[\mathrm{TE}]=$ $30 \mathrm{msec}$, flip angle $=90^{\circ}$ ). For each participant, around 620 functional volumes were acquired, and the first three volumes were discarded to avoid $\mathrm{T}_{1}$ saturation effects. After the EPI acquisition, a gradient-recalled sequence was applied to acquire two complex images with different TEs (TE $=4.92$ and $7.38 \mathrm{msec}$, respectively; TR $=367 \mathrm{msec}$, $\mathrm{FoV}=230 \times 230 \mathrm{~mm}^{2}, 64 \times 64$ matrix, 34 transverse slices with $3 \mathrm{~mm}$ thickness and 25\% interslice gap, flip angle = $90^{\circ}$, bandwidth $=260 \mathrm{~Hz} /$ pixel) and generate field maps for distortion correction of the EPI images. A structural MR scan was obtained at the end of the session ( $T_{1}$-weighted 3-D MP-RAGE sequence, $\mathrm{TR}=1960 \mathrm{msec}$, TE $=4.4 \mathrm{msec}$, FoV $=230 \times 173 \mathrm{~mm}^{2}$, matrix size $256 \times 192 \times 176$, voxel size $0.9 \times 0.9 \times 0.9 \mathrm{~mm}^{3}$ ).

\section{fMRI Data Analysis}

Data were preprocessed and analyzed using SPM8 (Wellcome Department of Imaging Neuroscience, http//www.fil.ion. ucl.ac.uk/spm). EPI time series were corrected for motion and distortion using Realign and Unwarp (Andersson, Hutton, Ashburner, Turner, \& Friston, 2001) together with the Fieldmap Toolbox (Hutton et al., 2002). The mean realigned EPI image was coregistered to the structural T1 image, and the coregistration parameters were applied to the realigned EPI time series. The T1 image was segmented into gray matter, white matter, and cerebrospinal fluid, using the unified segmentation approach (Ashburner \& Friston, 2005), and the coregistered functional images were normalized to MNI space (voxel size: $2 \times 2 \times$ $2 \mathrm{~mm}^{3}$ ) using the normalization parameters obtained from the segmentation procedure. Finally, the functional images were smoothed with a Gaussian kernel with FWHM of $8 \mathrm{~mm}$.

Preprocessed fMRI data were analyzed using the general linear model. For each participant, the task phase (onset $=$ presentation of the event pair; duration $=6 \mathrm{sec}$ ) was modeled separately for each condition (i.e., past order, future order, past event, future event) and convolved with the canonical hemodynamic response function to create regressors of interest. RTs were entered in the model as linear parametric regressors to ensure that differences between conditions are not simply due to differences in RTs (see Behavioral Results). The design matrix also included the realignment parameters to account for any residual movement-related effect. The canonical hemo- dynamic response function was used and a high-pass filter was implemented using a cutoff period of $128 \mathrm{sec}$ to remove the low-frequency drifts from the time series. Serial autocorrelations were estimated with a restricted maximum likelihood algorithm with an autoregressive model of order 1 ( + white noise).

For each participant, four contrasts of interest corresponding to the effect of each condition were computed. The individual contrast images were then entered into a second-level random-effects analysis using a two-way repeated-measures ANOVA. This generated a statistical parametric map of $F$ values for the main effect of Temporal Orientation (past vs. future), the main effect of Task (order vs. event), and the interaction between the two factors; $t$ contrasts were then performed to examine the direction of effects. In addition, to identify brain regions that were commonly recruited when processing temporal information for past and future events, we carried out a conjunction analysis (testing the conjunction null hypothesis; Friston, Penny, \& Glaser, 2005) of the order > event contrast for past events and the order $>$ event contrast for future events.

We also examined changes in the neural correlates of temporal order judgments as a function of the temporal distance between the two events constituting the event pairs. For each participant, we estimated a general linear model that included regressors for the task phase of each condition (i.e., past order, future order, past event, future event), linear parametric regressors coding for RTs, two linear parametric regressors indexing time lag (i.e., the number of days separating the two events of a pair, which ranged from 1 to 6 ) for the past and future order conditions, and the realignment parameters. Subsequently, random-effects analyses were performed on the parameter estimates of the parametric regressor for temporal lag in the past and future order conditions separately, using one-sample $t$ tests. To ensure that the regions that were correlated with temporal lag were indeed recruited for making temporal order judgments, the conditions of interest were inclusively masked (at $p<.001$, uncorrected) with the main effect of Task (order $>$ event) identified in the two-way ANOVA described above.

We report activations that were statistically significant at the .05 level, corrected for multiple comparisons (familywise error, FWE) at the voxel level over the entire brain volume. For our a priori ROIs in the hippocampus, lateral pFC, and parietal cortex, we used small volume correction $(p<.05$, FWE-corrected $)$ within anatomically defined ROIs. ROIs corresponding to the bilateral hippocampus (Amunts et al., 2005) and bilateral intraparietal sulcus (IPS; Scheperjans, Eickhoff, et al., 2008; Scheperjans, Hermann, et al., 2008; Choi et al., 2006) were created using the SPM Anatomy Toolbox v1.8 (Eickhoff et al., 2005), and an ROI including the bilateral middle frontal gyrus was created using the Wake Forest University PickAtlas Utility v3.0 (Maldjian, Laurienti, Kraft, \& Burdette, 2003). 


\section{RESULTS}

\section{Behavioral Results}

We first examined whether judgment accuracy and RTs differed across the four conditions. Correct responses for order judgments were calculated using the dates provided for each event during the prescan interview. For event judgments, correct responses corresponded to the identification of the events that were part of the participants' personal past or future. A 2 (Task: order vs. event) $\times 2$ (Temporal Orientation: past vs. future) repeatedmeasures ANOVA on correct responses showed that accuracy was significantly higher for event judgments than for order judgments, $F(1,21)=40.58, p<.001, \eta_{\mathrm{p}}{ }^{2}=0.66$; the main effect of Temporal Orientation and the interaction were not significant, $F(1,21)=1.64, p=.21, \eta_{\mathrm{p}}{ }^{2}=$ 0.07 , and $F(1,21)=1.04, p=.32, \eta_{\mathrm{p}}{ }^{2}=0.05$, respectively (see Table 1 for means and $S D$ s). For both past and future events, correct order judgments were significantly higher than chance (i.e., higher than 0.50), $t(21)=15.03, p<$ .001 , and $t(21)=15.10, p<.001$, respectively. A two-way ANOVA on RTs also revealed a significant effect of Task, showing that responses were faster for event judgments than for order judgments, $F(1,21)=294.45, p<.001$, $\eta_{\mathrm{p}}{ }^{2}=0.93$; the main effect of Temporal Orientation and the interaction were not significant, $F(1,21)=0.69, p=$ $.42, \eta_{\mathrm{p}}^{2}=0.03$, and $F(1,21)=2.03, p=.17, \eta_{\mathrm{p}}^{2}=0.09$, respectively (see Table 1 for means and $S D$ s).

Next, we examined whether order judgments were affected by the temporal distance between the two events of a pair. The number of days separating the two events (which ranged from 1 to 6) was used as a predictor variable in a regression model with RT as dependent variable. An interaction term was also included in the model to investigate whether the effect of temporal distance differed across temporal orientations (past vs. future). To account for the hierarchical structure of the data (i.e., trials are nested within participants and are thus not independent), we used multilevel modeling (Goldstein, 2011) with trials (event pairs) as level 1 units and participants as level 2 units. A random intercept multilevel model yielded a significant effect of temporal distance, indicating that RT was longer when the time

Table 1. Mean Proportion of Correct Responses and Mean RT as a Function of Task (Order vs. Event) and Temporal Orientation (Past vs. Future)

\begin{tabular}{lccccc}
\hline & \multicolumn{2}{c}{ Order } & & \multicolumn{2}{c}{ Event } \\
\cline { 2 - 3 } \cline { 5 - 6 } & Past & Future & & Past & Future \\
\hline $\begin{array}{l}\text { Correct } \\
\text { responses }\end{array}$ & $0.85(0.11)$ & $0.87(0.12)$ & $0.99(0.02)$ & $0.99(0.02)$ \\
RT (in msec) & $3479(550)$ & $3371(469)$ & $2240(363)$ & $2267(406)$ \\
\hline
\end{tabular}

Standard deviations are provided in parentheses.
Table 2. Brain Regions Showing Increased Activity for Order versus Event Judgments

\begin{tabular}{|c|c|c|c|c|c|c|}
\hline \multirow[b]{2}{*}{ Brain Region } & \multirow[b]{2}{*}{ Side } & \multicolumn{3}{|c|}{ MNI Coordinates } & \multirow{2}{*}{$\begin{array}{c}\text { Cluster } \\
\text { Size }\end{array}$} & \multirow[b]{2}{*}{$t$} \\
\hline & & $x$ & $y$ & $z$ & & \\
\hline \multirow[t]{5}{*}{ IPS } & \multirow[t]{3}{*}{$\mathrm{L}$} & -30 & -60 & 42 & 655 & 7.69 \\
\hline & & -32 & -48 & 34 & same & 6.49 \\
\hline & & -30 & -76 & 32 & same & 6.32 \\
\hline & \multirow[t]{2}{*}{$\mathrm{R}$} & 36 & -60 & 32 & 723 & 6.88 \\
\hline & & 44 & -44 & 48 & same & 6.48 \\
\hline \multirow[t]{3}{*}{ Visual cortex } & $\mathrm{R}$ & 20 & -58 & 18 & 1150 & 7.63 \\
\hline & \multirow[t]{2}{*}{$\mathrm{L}$} & -10 & -78 & 2 & same & 6.87 \\
\hline & & -16 & -62 & 20 & 88 & 6.02 \\
\hline \multirow[t]{2}{*}{ Anterior insula } & $\mathrm{R}$ & 30 & 22 & 2 & 337 & 7.55 \\
\hline & $\mathrm{L}$ & -30 & 24 & -2 & 159 & 6.83 \\
\hline \multirow[t]{2}{*}{ Dorsal ACC/pre-SMA } & \multirow[t]{2}{*}{ M } & 4 & 22 & 42 & 436 & 7.28 \\
\hline & & 8 & 28 & 30 & same & 5.57 \\
\hline \multirow[t]{2}{*}{ Precuneus } & M & 10 & -68 & 42 & 568 & 7.28 \\
\hline & M & -14 & -66 & 36 & same & 6.52 \\
\hline \multirow[t]{3}{*}{ Dorsolateral pFC } & \multirow[t]{2}{*}{$\mathrm{R}$} & 30 & 12 & 58 & 166 & 6.86 \\
\hline & & 48 & 36 & 26 & 147 & 6.74 \\
\hline & $\mathrm{L}$ & -42 & 26 & 24 & 7 & 5.24 \\
\hline \multirow[t]{2}{*}{ Rostrolateral pFC } & \multirow[t]{2}{*}{$\mathrm{R}$} & 36 & 56 & -2 & 24 & 5.79 \\
\hline & & 26 & 60 & 0 & 28 & 5.31 \\
\hline Cerebellum & $\mathrm{L}$ & -32 & -66 & -36 & 16 & 5.75 \\
\hline Precentral gyrus & $\mathrm{L}$ & -32 & 2 & 58 & 63 & 5.43 \\
\hline
\end{tabular}

All regions are significant at $p<.05$, corrected for multiple comparisons (FWE) at the voxel level over the entire brain volume. $\mathrm{L}$ and $\mathrm{R}$ refer to the left and right hemisphere, respectively. M refers to medial clusters. For large clusters, local maxima that are more than $10 \mathrm{~mm}$ apart are reported; "same" indicates that these local maxima are part of the same cluster as the peak reported above.

separating the two events decreased (coefficient $=$ $-52.98, S E=18.35, Z=2.89, p=.004)$. The interaction term was not significant (coefficient $=-19.28, S E=16.03$, $Z=1.20, p=.23$ ), indicating that the effect of temporal distance on RT was similar for past and future events.

\section{fMRI Results}

The brain regions involved in processing temporal order in past and future thought were investigated using a 2 (Task: order vs. event) $\times 2$ (Temporal Orientation: past vs. future) repeated-measures ANOVA. This analysis revealed a main effect of Task in several brain regions. On the other hand, no brain region showed a main effect of Temporal Orientation or an interaction between Task and Temporal Orientation. 


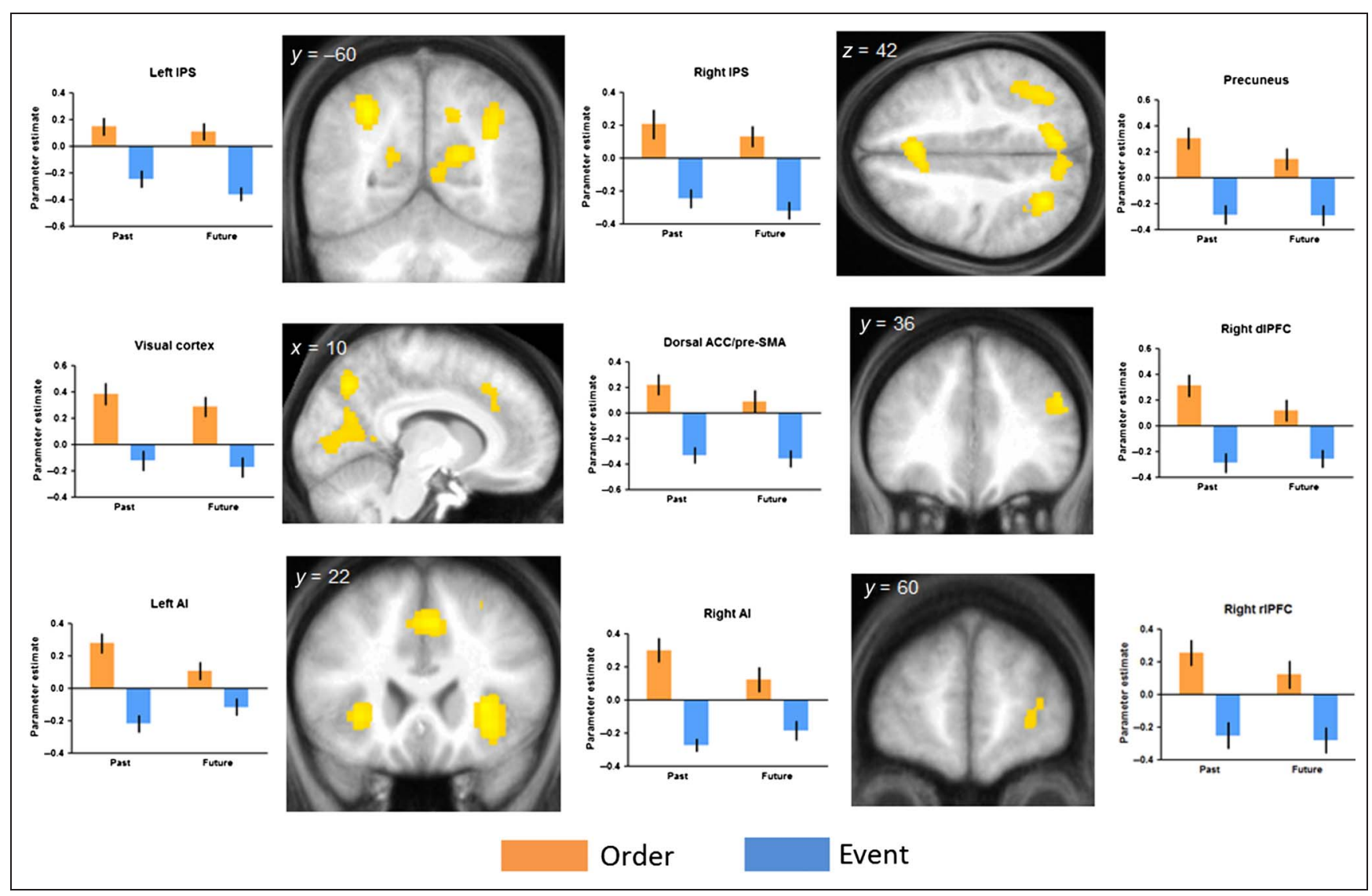

Figure 1. Brain regions showing increased activity for order versus event judgments. Displayed at $p<.05$ (FWE-corrected) on the mean structural MRI of all participants. Bar graphs show the mean parameter estimates for each condition (error bars represent $S E M$ ). dlPFC $=$ dorsolateral $\mathrm{pFC}$; $\mathrm{rlPFC}=$ rostrolateral $\mathrm{pFC} ; \mathrm{AI}=$ anterior insula.

To specify the main effect of Task, we first investigated regions that were significantly more activated when participants thought about the temporal order of past or future events compared with when they thought about the content of these events. A $t$ contrast revealed increased activation in the IPS bilaterally (along the entire anterior-posterior axis of the IPS), lateral pFC (in the dorsolateral $\mathrm{pFC}$ bilaterally and in the right rostrolateral pFC), dorsal ACC/pre-SMA, anterior insula bilaterally, precuneus, and visual cortex (Table 2, Figure 1). We did not find significant activation in the hippocampus after whole-brain correction for multiple comparisons $(p<$ .05 , FWE-corrected), but FWE correction within an anatomically defined hippocampal ROI (see Methods) revealed voxels in the left posterior hippocampus that showed higher activation when processing temporal order (peak MNI coordinate: $x=-24, y=-36, z=6 ; t=$ $4.29, p=.02$; cluster size: 24 voxels; Figure 2 ). The reverse $t$ contrast revealed a number of brain regions that were significantly more activated when participants thought about the content of past and future events compared with when they thought about their temporal order, including the lateral temporal cortex bilaterally, medial prefrontal regions (medial $\mathrm{OFC}$ and dorsomedial $\mathrm{pFC}$ ), and anterior hippocampus bilaterally (see Table 3, Figure 3). Because the proportion of correct responses differed between the order and event conditions (see Behavioral Results), we also rerun the same analyses using only trials for which participants provided a correct response. All results reported above remained unchanged.

To further identify brain regions that were commonly recruited when processing the order of past and future events, we carried out a conjunction analysis (testing the conjunction null hypothesis) of the order $>$ event contrast for past events and the order $>$ event contrast

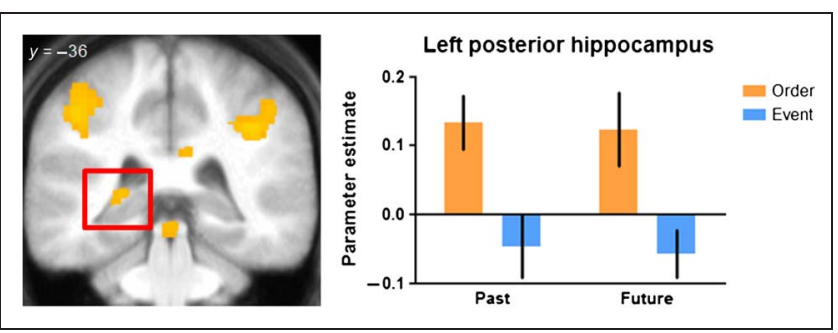

Figure 2. Area of the left posterior hippocampus showing increased activity for order versus event judgments. Displayed at $p<.001$ (uncorrected) on the mean structural MRI of all participants. Bar graphs show the mean parameter estimates for each condition (error bars represent $S E M$ ). 
Table 3. Brain Regions Showing Increased Activity for Event versus Order Judgments

\begin{tabular}{|c|c|c|c|c|c|c|}
\hline \multirow[b]{2}{*}{ Brain Region } & \multirow[b]{2}{*}{ Side } & \multicolumn{3}{|c|}{$\begin{array}{c}\text { MNI } \\
\text { Coordinates }\end{array}$} & \multirow{2}{*}{$\begin{array}{c}\text { Cluster } \\
\text { Size }\end{array}$} & \multirow[b]{2}{*}{$t$} \\
\hline & & $x$ & $y$ & $z$ & & \\
\hline \multirow[t]{3}{*}{ Middle temporal gyrus } & $\mathrm{R}$ & 60 & -60 & 10 & 195 & 6.97 \\
\hline & $\mathrm{L}$ & -60 & -64 & 6 & 66 & 5.75 \\
\hline & & -64 & -14 & -18 & 5 & 5.39 \\
\hline \multirow[t]{2}{*}{ Inferior temporal gyrus } & $\mathrm{R}$ & 36 & 16 & -36 & 4 & 5.32 \\
\hline & $\mathrm{L}$ & -46 & -2 & -40 & 4 & 5.24 \\
\hline Medial OFC & M & -2 & 44 & -22 & 140 & 6.68 \\
\hline \multirow[t]{2}{*}{ Anterior hippocampus } & $\mathrm{R}$ & 20 & -10 & -18 & 64 & 6.47 \\
\hline & $\mathrm{L}$ & -22 & -8 & -26 & 3 & 5.04 \\
\hline \multirow[t]{3}{*}{ Dorsal MPFC } & M & -6 & 58 & 18 & 207 & 6.12 \\
\hline & & 8 & 60 & 20 & same & 6.00 \\
\hline & & -10 & 52 & 38 & 18 & 5.74 \\
\hline Postcentral gyrus & $\mathrm{R}$ & 28 & -32 & 70 & 34 & 6.11 \\
\hline Supramarginal gyrus & $\mathrm{R}$ & 66 & -32 & 28 & 63 & 5.94 \\
\hline Middle cingulate cortex & M & 6 & -16 & 44 & 17 & 5.36 \\
\hline
\end{tabular}

All regions are significant at $p<.05$, corrected for multiple comparisons (FWE) at the voxel level over the entire brain volume. $\mathrm{L}$ and $\mathrm{R}$ refer to the left and right hemisphere, respectively. M refers to medial clusters. For large clusters, local maxima that are more than $10 \mathrm{~mm}$ apart are reported; "same" indicates that these local maxima are part of the same cluster as the peak reported above.

for future events. This revealed common activations in the IPS bilaterally, dorsal ACC/pre-SMA, right dorsolateral pFC, visual cortex, and left posterior hippocampus (Table 4). The other regions that were associated with the main effect of task (order $>$ event) in the two-way ANOVA (i.e., the anterior insula, right rostrolateral $\mathrm{pFC}$, and precuneus) did not survive whole-brain correction for multiple comparisons ( $p<.05$, FWE-corrected) in the conjunction analysis but were all significant at a relaxed threshold $(p<$ .001 , uncorrected). A direct contrast between the past and future order conditions did not reveal any significant difference.

Next, we examined whether the brain regions recruited for determining the order of past and future events were modulated by the temporal distance separating the two events of a pair. To identify regions where activity monotonically increased or decreased as a function of temporal distance, we conducted parametric modulation analyses with the number of days separating the events as linear parametric modulator. This parametric analysis was conducted separately for past and future events and the contrast of interests were inclusively masked with the main effect of Task (order > event) identified in the two-way ANOVA reported above to ensure that the identified regions were involved in temporal order processing. No brain regions showed a significant positive or negative correlation with time lag at $p<.05$, FWE-corrected over the entire brain volume. However, further analyses using small volume correction within anatomically defined ROIs (see Methods) identified a region of the right posterior IPS showing a negative correlation with time lag in the past order condition, suggesting that the right posterior IPS was recruited to a greater extent for processing the order of past events that were closer to each other in time (peak MNI coordinate: $x=36, y=-64, z=52 ; t=$ $5.01, p=.03$; cluster size: 6 voxels; Figure $4 \mathrm{~A}$ ); there was no significant correlation between time lag and activity within the hippocampal and prefrontal ROIs. For future events, there was no significant correlation between time lag and activity within the selected ROIs, but an exploratory analysis at a relaxed threshold $(p<.001$, uncorrected, with a minimum of five contiguous voxels) revealed a region of the left posterior IPS showing a negative correlation with time lag (peak MNI coordinate: $x=-30, y=-68$, $z=36 ; t=4.09$; cluster size: 12 voxels; Figure 4B).

\section{DISCUSSION}

Although considerable progress has recently been made in elucidating how autobiographical thoughts are constructed and elaborated (Mullally \& Maguire, 2014; D’Argembeau, 2012; Schacter et al., 2012), relatively little is known about their temporal dimension, especially with respect to the future (Klein, 2013; Szpunar, 2011; Friedman, 2005). Here we focused on the event-sequencing aspect of time and investigated whether similar or distinct neurocognitive processes are involved in ordering past events and planned future events. Our findings revealed important commonalities in temporal order processing for past and future events, both in terms of behavioral performance and regional brain activation. Behavioral results showed that judgments of temporal order were above chance for both past and future events (with no significant difference in performance between the two kinds of events), demonstrating that coherent knowledge about the order of personal events can be formed not only for the past (in line with previous findings; St Jacques et al., 2008; Skowronski et al., 2003), but also for the future. Moreover, we found that the temporal distance between the two events of a pair influenced order judgments in a similar way for past and future events. The fMRI data indicated that the same brain regions were recruited for determining the order of past and future events, with no significant difference between the two kinds of events. Collectively, these findings suggest that the same processing operations were engaged for ordering past events and planned future events in time.

Although neuroimaging studies have repeatedly shown hippocampal activations when people remember past events or imagine future events (e.g., Addis, Wong, \& Schacter, 2007; Okuda et al., 2003; for a recent meta-analysis, see Viard, Desgranges, Eustache, \& Piolino, 2012), the question 
Figure 3. Brain regions showing increased activity for event versus order judgments. Displayed at $p<.05$ (FWEcorrected) on the mean structural MRI of all participants. Bar graphs show the mean parameter estimates for each condition (error bars represent $S E M)$. mOFC $=$ medial OFC; $\mathrm{dmPFC}=$ dorsomedial $\mathrm{pFC}$; MTG $=$ middle temporal gyrus .

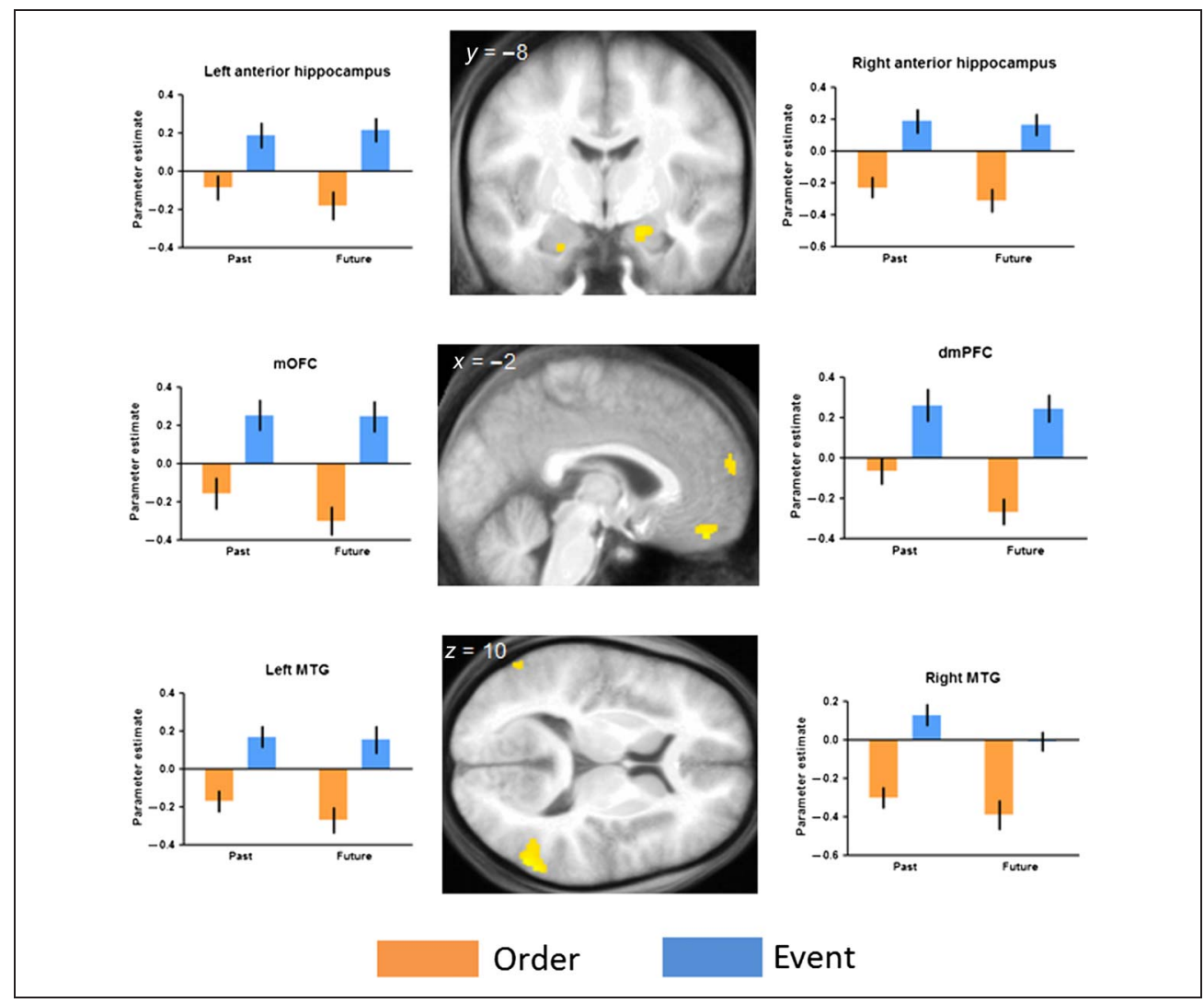

of whether the hippocampus contributes to locating events in time is still debated. On the one hand, there is evidence that the hippocampus is activated when imagining events or scenes that are not located at particular times in the past or future (Summerfield et al., 2010; Hassabis et al., 2007), and a recent study failed to detect significant hippocampal activity when participants placed an imagined event in the

Table 4. Brain Regions Showing Increased Activity for Both Past and Future Order Judgments (Conjunction Analysis)

\begin{tabular}{|c|c|c|c|c|c|c|}
\hline \multirow[b]{2}{*}{ Brain Region } & \multirow[b]{2}{*}{ Side } & \multicolumn{3}{|c|}{$\begin{array}{c}\text { MNI } \\
\text { Coordinates }\end{array}$} & \multirow{2}{*}{$\begin{array}{c}\text { Cluster } \\
\text { Size }\end{array}$} & \multirow[b]{2}{*}{$t$} \\
\hline & & $x$ & $y$ & $z$ & & \\
\hline \multirow[t]{2}{*}{ IPS } & $\mathrm{L}$ & -30 & -60 & 42 & 31 & $5.58^{\mathrm{a}}$ \\
\hline & $\mathrm{R}$ & 38 & -60 & 32 & 18 & $5.35^{\mathrm{a}}$ \\
\hline Visual cortex & $\mathrm{L}$ & -10 & -78 & 2 & 21 & $5.45^{\mathrm{a}}$ \\
\hline Dorsal ACC/pre-SMA & M & 6 & 22 & 42 & 47 & $5.42^{\mathrm{a}}$ \\
\hline Dorsolateral pFC & $\mathrm{R}$ & 32 & 12 & 60 & 14 & $5.40^{\mathrm{a}}$ \\
\hline Posterior hippocampus & $\mathrm{L}$ & -24 & -36 & 4 & 2 & $3.30^{\mathrm{b}}$ \\
\hline
\end{tabular}

$\mathrm{L}$ and $\mathrm{R}$ refer to the left and right hemisphere, respectively. $\mathrm{M}$ refers to medial clusters.

${ }^{a}$ Significant at $p<.05$, corrected for multiple comparisons (FWE) at the voxel level over the entire brain volume.

${ }^{\mathrm{b}}$ Significant at $p<.05$, corrected for multiple comparisons (FWE) using small volume correction (see Methods).

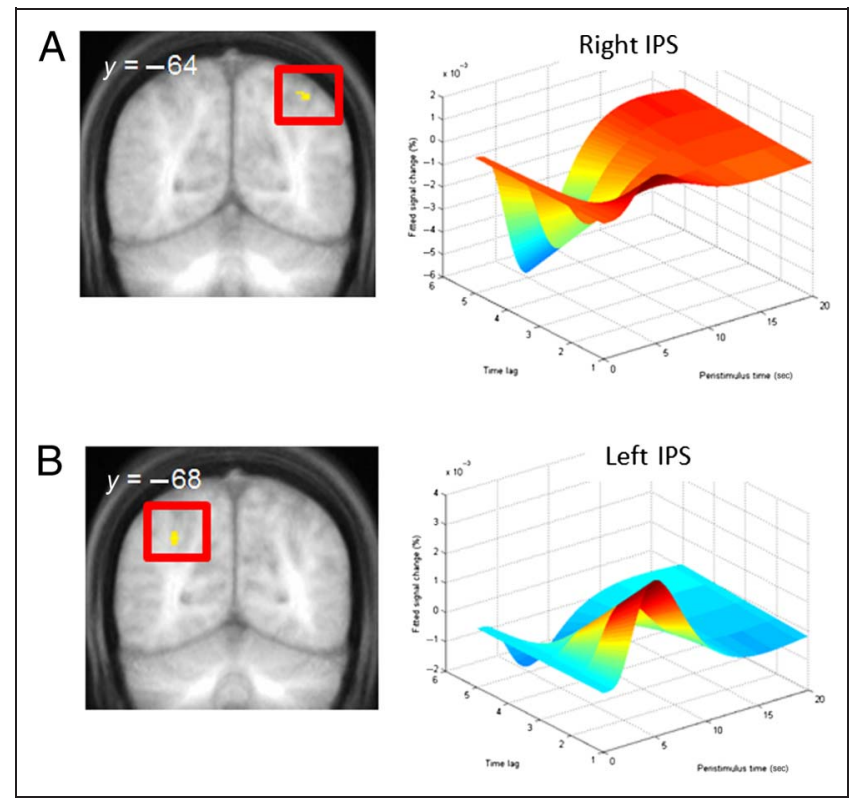

Figure 4. Brain regions showing a temporal distance effect during order judgments. (A) The right IPS showed greater activity when processing the order of past events that were closer to each other in time. (B) The left IPS showed greater activity when processing the order of future events that were closer to each other in time. Displayed at $p<.001$ (uncorrected) on the mean structural MRI of all participants. The three-dimensional graphs represent parametric plots of the group average of each participant's fitted data. 
past or future relative to a condition in which they imagined the same event occurring in the present moment (Nyberg et al., 2010). These findings have led to the view that the hippocampus contributes to event representation (i.e., the construction of spatially coherent scenes) rather than temporal processes (Mullally \& Maguire, 2014; Eacott \& Easton, 2012; Hassabis \& Maguire, 2007). On the other hand, studies that specifically focused on the eventsequencing aspect of time demonstrated hippocampal activations during the retrieval of temporal order (Ekstrom et al., 2011; Lehn et al., 2009), suggesting that the hippocampus plays a role in the temporal organization of event representations (Eichenbaum, 2013).

Our finding that event content and temporal order were both associated with hippocampal activations, but in distinct portions, may help reconcile these two views on the contribution of the hippocampus to past and future thought. Indeed, our data suggest a dissociation between anterior and posterior hippocampal regions in processing different aspects of autobiographical thought, with the anterior hippocampus being involved in representing the content of past and future events and the posterior hippocampus in determining their temporal order. ${ }^{1}$ This distinction is consistent with a recent model of long-axis hippocampal specialization, whereby the anterior and posterior hippocampus represent information at coarse and fine granularities, respectively (Poppenk, Evensmoen, Moscovitch, \& Nadel, 2013). Applying this model to autobiographical thought, Poppenk et al. (2013) proposed that the anterior hippocampus might support gist-based representations of event content (by forming associative links between the principal actors, actions, and setting of an event), whereas the posterior hippocampus might represent more specific contextual details, including temporal information. The left posterior hippocampal region observed here could thus contribute to establish temporal order in past and future thought either by representing temporal information itself or by representing other contextual details that are used to infer temporal information (see Friedman, 1993, for further discussion of how contextual information associated with an event can be used to infer its location in time). In line with this view, it has been shown that activity within the left posterior hippocampus correlates with the amount of details experienced when representing past and future events (Addis \& Schacter, 2008; see also Viard et al., 2012).

Another important finding of this study is that many brain regions that were activated when thinking about temporal order-notably the IPS, lateral pFC, dorsal ACC/pre-SMA, and anterior insula-lied outside the core network that has been associated with autobiographical memory and future thought (Mullally \& Maguire, 2014; Martinelli, Sperduti, \& Piolino, 2013; Kim, 2012; Schacter et al., 2012; McDermott, Szpunar, \& Christ, 2009; Spreng, Mar, \& Kim, 2009). These regions are part of the frontoparietal and cingulo-opercular networks that have been linked to cognitive control (Power \& Petersen, 2013;
Dosenbach, Fair, Cohen, Schlaggar, \& Petersen, 2008; Vincent, Kahn, Snyder, Raichle, \& Buckner, 2008), which supports the idea that temporal information (or at least temporal order) is not necessarily an intrinsic property of memory or future thought but is often determined using additional, effortful processes (Friedman, 1993, 2005). Interestingly, recent studies have shown that autobiographical planning and goal-directed simulation (i.e., the strategic formulation of plans to reach desired goal states) engage not only the core network involved in past and future thought, but also the frontoparietal control network (Gerlach, Spreng, Madore, \& Schacter, in press; Gerlach, Spreng, Gilmore, \& Schacter, 2011; Spreng, Stevens, Chamberlain, Gilmore, \& Schacter, 2010). Insofar as the ability to order events in time is a key component of planning and goal pursuit, our findings align nicely with these studies and further suggest that the frontoparietal control network plays a role in the temporal organization of the planning process (e.g., sequencing events in time).

The frontal components of the frontoparietal network, as well as other prefrontal areas, have been linked to the processing of temporal order for past events (e.g., Ekstrom et al., 2011; St Jacques et al., 2008; Fujii et al., 2004), and our data show that the same prefrontal regions are involved in ordering planned future events, providing additional support to the general view that the lateral $\mathrm{pFC}$ contributes to the temporal organization of cognition and behavior (Fuster, 2001; Ingvar, 1985). In line with our predictions, we also found that the parietal cortex and, more specifically, the IPS contribute to order processing in autobiographical thought. One interpretation of this finding is that people use spatial representations to order past and future events in time and that the IPS supports such time-space mappings (Bueti \& Walsh, 2009). There is indeed substantial evidence that time is construed in terms of space (e.g., Nunez \& Cooperrider, 2013; Christian et al., 2012; Arzy, Adi-Japha, et al., 2009; Boroditsky, 2000), and research has shown that visuospatial imagery contributes to the representation of time patterns of relevance to this study, such as the days of the week (Friedman, 2005). The view that visuospatial processes contributes to temporal order judgments is further supported by our finding that these judgments also engaged the visual cortex and precuneus (more specifically a dorsal-posterior section of the precuneus that has been associated with visuospatial imagery; Zhang \& Li, 2012). Indeed, when considered as a whole, the brain regions that were activated during temporal order judgments-the IPS, precuneus, occipital cortex, and lateral pFC_-closely correspond to the neural network that has been linked to visuospatial imagery (Sack \& Schuhmann, 2012).

The present results further showed that the IPS was recruited to a greater extent when the events people had to order were closer to each other in time. A possible interpretation of this finding is that, with decreasing time lag between events, it is more difficult to discriminate the respective locations of events on a mental time line, thus 
placing higher demands on the IPS. Another possible interpretation of the effect of temporal distance on IPS activity draws on the idea that the IPS may play a general role in ordinal processing. Distance effects in IPS activity have indeed been observed in many different domains, including numerical and alphabetical processing (Fias, Lammertyn, Caessens, \& Orban, 2007; Pinel, Dehaene, Riviere, \& LeBihan, 2001), and STM for order (Attout, Fias, Salmon, \& Majerus, 2014; Marshuetz, Reuter-Lorenz, Smith, Jonides, \& Noll, 2006). These and related findings have led to the view that the IPS (especially the anterior IPS) supports domain general ordinal processes that allow coding for serial order in various task domains (Attout et al., 2014). As time frames such as the days of the week contain an inherently ordinal structure, it could be that the distance effect in IPS activity observed here reflects the use of ordinal processing to determine the relative order of events. It should be noted, however, that the location of IPS activation we detected in this study is posterior to the region that has been typically associated with ordinal processing, although some studies have demonstrated distance effects in the posterior IPS as well (e.g., Pinel et al., 2001).

When examining the brain regions associated with representing the content of past and future events, we detected activation in some but not all regions of the core network that has been associated with autobiographical thought (Schacter et al., 2012). Notably, we did not find differential activation in two central regions of the core network: the anterior medial $\mathrm{pFC}$ and posterior cingulate cortex (Andrews-Hanna, Reidler, Sepulcre, Poulin, \& Buckner, 2010). This is likely because of the nature of the comparison task: In this study, representing the content of past and future events was compared with a task that also involved personal events (the temporal order task), whereas previous studies that have shown activation of the entire core network compared the representation of past and future events with control tasks that involved the retrieval of nonpersonal information from semantic memory or the imagination of nonpersonal events (e.g., Botzung, Denkova, \& Manning, 2008; Addis et al., 2007; Szpunar, Watson, \& McDermott, 2007). Midline cortical regions such as the medial $\mathrm{pFC}$ may contribute to represent the personal significance of past and future events (Andrews-Hanna et al., 2010; D'Argembeau et al., 2010), a process that was likely subtracted out in the current study as the same personal events were used in the two conditions of interest.

Although the present behavioral and neuroimaging results suggest that common cognitive processes are involved in ordering past events and planned future events in time, the precise nature of these processes remains to be fully specified. One possibility is that people locate events within known time patterns (such as the parts of the day, week, and year), and as already discussed, this process may in part depend on visuospatial imagery (Friedman, 2005). Another, not incompatible but per- haps additional, process may consist in retrieving order codes to make order judgments (Friedman, 1993). For past events, an order code may be created when a particular event reminds a previous event and information about the relative order of the two events is encoded in and thus can later be retrieved from memory. A similar case can be made for future events: When imagining a future event, one may remember another related future event that one has previously envisioned and an order code that links the two events may be established in memory. Indeed, recent findings have shown that both past and future events are frequently part of event clusters that link a series of events together and organize them in causal sequences (D’Argembeau \& Demblon, 2012). Finally, people could in part base their judgments on feelings of subjective distance (D'Argembeau \& Van der Linden, 2012; Friedman, 1993), and for a few events, exact dates might be available (Friedman, 1993, 2005).

As a final point, it should be noted that this study investigated temporal order processing in future thoughts that have already been established in memory-referred to as "memories of the future" (Szpunar, Addis, McLelland, \& Schacter, 2013; Ingvar, 1985). Indeed, the future events that were presented to participants in the scanner had already been imagined on a previous occasion and were associated with specific temporal locations. Another important question that would be worthy of consideration in future studies is how the temporal location of a future event is established in the first place (i.e., when imagining this event for the first time). Elucidating this question may be key in understanding what distinguishes future thoughts from other types of imagined events—events that can be vividly imagined but are not accompanied by the feeling that they will actually occur in one's personal future (de Vito, Gamboz, \& Brandimonte, 2012). An intriguing possibility is that an imagined event may become truly autobiographical -in the sense of being associated with a sense of personal plausibility (Scoboria, Mazzoni, Kirsch, \& Relyea, 2004)— only when it acquires a temporal context by virtue of its integration in higher-order event sequences that are established for goal pursuit (D’Argembeau \& Demblon, 2012).

\section{Acknowledgments}

This work was supported by the French-speaking community of Belgium (ARC, Convention 12/17-01-REST) and the National Fund for Scientific Research (FRS-FNRS), Belgium. A. D., S. M., and C. B. are Research Associates of the FRS-FNRS.

Reprint requests should be sent to Arnaud D'Argembeau, Department of Psychology-Cognition \& Behavior, University of Liège, Boulevard du Rectorat 3 (B33), 4000 Liège, Belgium, or via e-mail: a.dargembeau@ulg.ac.be.

\section{Note}

1. Another possible interpretation of the activation of the anterior hippocampus observed in this study would be that it reflects differences in novelty signals between conditions (e.g., 
Kafkas \& Montaldi, 2014). Indeed, it could be argued that the two conditions involved different amount of stimulus novelty because the temporal ordering task included two events that had been generated during the prescan interview, whereas the event task contained one event that had been generated in the prescan interview and one event taken from another person. Although this interpretation in terms of stimulus novelty cannot be totally excluded, we believe it is unlikely for two reasons. First, the nonpersonal events that were included in the event task were repeated three times throughout the fMRI session, thus minimizing possible stimulus novelty effects. Second, participants were instructed to focus on representing the content of the personal (i.e., more familiar) events.

\section{REFERENCES}

Addis, D. R., \& Schacter, D. L. (2008). Constructive episodic simulation: Temporal distance and detail of past and future events modulate hippocampal engagement. Hippocampus, 18, 227-237.

Addis, D. R., Wong, A. T., \& Schacter, D. L. (2007). Remembering the past and imagining the future: Common and distinct neural substrates during event construction and elaboration. Neuropsychologia, 45, 1363-1377.

Amunts, K., Kedo, O., Kindler, M., Pieperhoff, P., Mohlberg, H., Shah, N. J., et al. (2005). Cytoarchitectonic mapping of the human amygdala, hippocampal region and entorhinal cortex Intersubject variability and probability maps. Anatomy and Embryology, 210, 343-352.

Andersson, J. L., Hutton, C., Ashburner, J., Turner, R., \& Friston, K. (2001). Modeling geometric deformations in EPI time series. Neuroimage, 13, 903-919.

Andrews-Hanna, J. R., Reidler, J. S., Sepulcre, J., Poulin, R., \& Buckner, R. L. (2010). Functional-anatomic fractionation of the brain's default network. Neuron, 65, 550-562.

Arzy, S., Adi-Japha, E., \& Blanke, O. (2009). The mental time line: An analogue of the mental number line in the mapping of life events. Consciousness and Cognition, 18, 781-785.

Arzy, S., Collette, S., Ionta, S., Fornari, E., \& Blanke, O. (2009). Subjective mental time: The functional architecture of projecting the self to past and future. European Journal of Neuroscience, 30, 2009-2017.

Ashburner, J., \& Friston, K. J. (2005). Unified segmentation. Neuroimage, 26, 839-851.

Atance, C. M., \& O’Neill, D. K. (2001). Episodic future thinking. Trends in Cognitive Sciences, 5, 533-539.

Attout, L., Fias, W., Salmon, E., \& Majerus, S. (2014). Common neural substrates for ordinal representation in short-term memory, numerical and alphabetical cognition. PLoS One, 9, e92049.

Berntsen, D., \& Jacobsen, A. S. (2008). Involuntary (spontaneous) mental time travel into the past and future. Consciousness and Cognition, 17, 1093-1104.

Boroditsky, L. (2000). Metaphoric structuring: Understanding time through spatial metaphors. Cognition, 75, 1-28.

Botzung, A., Denkova, E., \& Manning, L. (2008). Experiencing past and future personal events: Functional neuroimaging evidence on the neural bases of mental time travel. Brain and Cognition, 66, 202-212.

Boyer, P. (2008). Evolutionary economics of mental time travel? Trends in Cognitive Sciences, 12, 219-224.

Buckner, R. L., \& Carroll, D. C. (2007). Self-projection and the brain. Trends in Cognitive Sciences, 11, 49-57.

Bueti, D., \& Walsh, V. (2009). The parietal cortex and the representation of time, space, number and other magnitudes. Philosophical Transactions of the Royal Society of London, Series B, Biological Sciences, 364, 1831-1840.

Choi, H. J., Zilles, K., Mohlberg, H., Schleicher, A., Fink, G. R., Armstrong, E., et al. (2006). Cytoarchitectonic identification and probabilistic mapping of two distinct areas within the anterior ventral bank of the human intraparietal sulcus. Journal of Comparative Neurology, 495, 53-69.

Christian, B. M., Miles, L. K., \& Macrae, C. N. (2012). Your space or mine? Mapping self in time. PLOS ONE, 7, e49228.

Conway, M. A. (2009). Episodic memories. Neuropsychologia, 47, 2305-2313.

D'Argembeau, A. (2012). Autobiographical memory and future thinking. In D. Berntsen \& D. C. Rubin (Eds.), Understanding autobiographical memory: Theories and approaches (pp. 311-330). Cambridge: Cambridge University Press.

D'Argembeau, A., \& Demblon, J. (2012). On the representational systems underlying prospection: Evidence from the eventcueing paradigm. Cognition, 125, 160-167.

D'Argembeau, A., Renaud, O., \& Van der Linden, M. (2011). Frequency, characteristics, and functions of future-oriented thoughts in daily life. Applied Cognitive Psychology, 25, 96-103.

D’Argembeau, A., Stawarczyk, D., Majerus, S., Collette, F., Van der Linden, M., Feyers, D., et al. (2010). The neural basis of personal goal processing when envisioning future events. Journal of Cognitive Neuroscience, 22, 1701-1713.

D'Argembeau, A., \& Van der Linden, M. (2012). Predicting the phenomenology of episodic future thoughts. Consciousness and Cognition, 21, 1198-1206.

de Vito, S., Gamboz, N., \& Brandimonte, M. A. (2012). What differentiates episodic future thinking from complex scene imagery? Consciousness and Cognition, 21, 813-823.

Dosenbach, N. U., Fair, D. A., Cohen, A. L., Schlaggar, B. L., \& Petersen, S. E. (2008). A dual-networks architecture of top-down control. Trends in Cognitive Sciences, 12, 99-105.

Eacott, M. J., \& Easton, A. (2012). Remembering the past and thinking about the future: Is it really about time? Learning and Motivation, 43, 200-208.

Eichenbaum, H. (2013). Memory on time. Trends in Cognitive Sciences, 17, 81-88.

Eickhoff, S. B., Stephan, K. E., Mohlberg, H., Grefkes, C., Fink, G. R., Amunts, K., et al. (2005). A new SPM toolbox for combining probabilistic cytoarchitectonic maps and functional imaging data. Neuroimage, 25, 1325-1335.

Ekstrom, A. D., Copara, M. S., Isham, E. A., Wang, W. C., \& Yonelinas, A. P. (2011). Dissociable networks involved in spatial and temporal order source retrieval. Neuroimage, 56, 1803-1813.

Fias, W., Lammertyn, J., Caessens, B., \& Orban, G. A. (2007). Processing of abstract ordinal knowledge in the horizontal segment of the intraparietal sulcus. Journal of Neuroscience, 27, 8952-8956.

Friedman, W. J. (1993). Memory for the time of past events. Psychological Bulletin, 113, 44-66.

Friedman, W. J. (2004). Time in autobiographical memory. Social Cognition, 22, 591-605.

Friedman, W. J. (2005). Developmental and cognitive perspectives on human's sense of the times of past and future events. Learning and Motivation, 36, 145-158.

Friston, K. J., Penny, W. D., \& Glaser, D. E. (2005). Conjunction revisited. Neuroimage, 25, 661-667.

Fujii, T., Suzuki, M., Okuda, J., Ohtake, H., Tanji, K., Yamaguchi, K., et al. (2004). Neural correlates of context memory with real-world events. Neuroimage, 21, 1596-1603.

Fuster, J. M. (2001). The prefrontal cortex-An update: Time is of the essence. Neuron, 30, 319-333. 
Gerlach, K. D., Spreng, R. N., Gilmore, A. W., \& Schacter, D. L. (2011). Solving future problems: Default network and executive activity associated with goal-directed mental simulations. Neuroimage, 55, 1816-1824.

Gerlach, K. D., Spreng, R. N., Madore, K. P., \& Schacter, D. L. (in press). Future planning: Default network activity couples with frontoparietal control network and reward-processing regions during process and outcome simulations. Social Cognitive and Affective Neuroscience.

Goldstein, H. (2011). Multilevel statistical models (4th ed.). Chichester, UK: Wiley.

Hassabis, D., Kumaran, D., \& Maguire, E. A. (2007). Using imagination to understand the neural basis of episodic memory. Journal of Neuroscience, 27, 14365-14374.

Hassabis, D., \& Maguire, E. A. (2007). Deconstructing episodic memory with construction. Trends in Cognitive Sciences, 11, 299-306.

Hutton, C., Bork, A., Josephs, O., Deichmann, R., Ashburner, J., \& Turner, R. (2002). Image distortion correction in fMRI: A quantitative evaluation. Neuroimage, 16, 217-240.

Ingvar, D. H. (1985). "Memory of the future": An essay on the temporal organization of conscious awareness. Human Neurobiology, 4, 127-136.

Kafkas, A., \& Montaldi, D. (2014). Two separate, but interacting, neural systems for familiarity and novelty detection: A dualroute mechanism. Hippocampus, 24, 516-527.

Kim, H. (2012). A dual-subsystem model of the brain's default network: Self-referential processing, memory retrieval processes, and autobiographical memory retrieval. Neuroimage, 61, 966-977.

Klein, S. B. (2013). The complex act of projecting oneself into the future. Wiley Interdisciplinary Reviews. Cognitive Science, 4, 63-79.

Klinger, E., \& Cox, W. M. (1987). Dimensions of thought flow in everyday life. Imagination, Cognition, and Personality, 7, 105-128.

Lehn, H., Steffenach, H. A., van Strien, N. M., Veltman, D. J., Witter, M. P., \& Haberg, A. K. (2009). A specific role of the human hippocampus in recall of temporal sequences. Journal of Neuroscience, 29, 3475-3484.

Maldjian, J. A., Laurienti, P. J., Kraft, R. A., \& Burdette, J. H. (2003). An automated method for neuroanatomic and cytoarchitectonic atlas-based interrogation of fMRI data sets. Neuroimage, 19, 1233-1239.

Marshuetz, C., Reuter-Lorenz, P. A., Smith, E. E., Jonides, J., \& Noll, D. C. (2006). Working memory for order and the parietal cortex: An event-related functional magnetic resonance imaging study. Neuroscience, 139, 311-316.

Martinelli, P., Sperduti, M., \& Piolino, P. (2013). Neural substrates of the self-memory system: New insights from a meta-analysis. Human Brain Mapping, 34, 1515-1529.

Mather, M., Cacioppo, J. T., \& Kanwisher, N. (2013). How fMRI can inform cognitive theories. Perspectives on Psychological Science, 8, 108-113.

McDermott, K. B., Szpunar, K. K., \& Christ, S. E. (2009). Laboratory-based and autobiographical retrieval tasks differ substantially in their neural substrates. Neuropsychologia, 47, 2290-2298.

Mullally, S. L., \& Maguire, E. A. (2014). Memory, imagination, and predicting the future: A common brain mechanism? Neuroscientist, 20, 220-234.

Nunez, R., \& Cooperrider, K. (2013). The tangle of space and time in human cognition. Trends in Cognitive Sciences, 17, 220-229.

Nyberg, L., Kim, A. S., Habib, R., Levine, B., \& Tulving, E. (2010). Consciousness of subjective time in the brain. Proceedings of the National Academy of Sciences, U.S.A., 107, 22356-22359.
Okuda, J., Fujii, T., Ohtake, H., Tsukiura, T., Tanji, K., Suzuki, K., et al. (2003). Thinking of the future and past: The roles of the frontal pole and the medial temporal lobes. Neuroimage, 19, 1369-1380.

Pinel, P., Dehaene, S., Riviere, D., \& LeBihan, D. (2001). Modulation of parietal activation by semantic distance in a number comparison task. Neuroimage, 14, 1013-1026.

Poppenk, J., Evensmoen, H. R., Moscovitch, M., \& Nadel, L. (2013). Long-axis specialization of the human hippocampus. Trends in Cognitive Sciences, 17, 230-240.

Power, J. D., \& Petersen, S. E. (2013). Control-related systems in the human brain. Current Opinion in Neurobiology, 23, 223-228.

Sack, A. T., \& Schuhmann, T. (2012). Hemispheric differences within the fronto-parietal network dynamics underlying spatial imagery. Frontiers in Psychology, 3, 214.

Schacter, D. L. (2012). Adaptive constructive processes and the future of memory. The American Psychologist, 67, 603-613.

Schacter, D. L., \& Addis, D. R. (2007). The cognitive neuroscience of constructive memory: Remembering the past and imagining the future. Philosophical Transactions of the Royal Society of London, Series B, Biological Sciences, 362, 773-786

Schacter, D. L., Addis, D. R., Hassabis, D., Martin, V. C., Spreng, R. N., \& Szpunar, K. K. (2012). The future of memory: Remembering, imagining, and the brain. Neuron, 76, 677-694.

Scheperjans, F., Eickhoff, S. B., Homke, L., Mohlberg, H., Hermann, K., Amunts, K., et al. (2008). Probabilistic maps, morphometry, and variability of cytoarchitectonic areas in the human superior parietal cortex. Cerebral Cortex, 18, 2141-2157.

Scheperjans, F., Hermann, K., Eickhoff, S. B., Amunts, K., Schleicher, A., \& Zilles, K. (2008). Observer-independent cytoarchitectonic mapping of the human superior parietal cortex. Cerebral Cortex, 18, 846-867.

Scoboria, A., Mazzoni, G., Kirsch, I., \& Relyea, M. (2004). Plausibility and belief in autobiographical memory. Applied Cognitive Psychology, 18, 791-807.

Skowronski, J. J., \& Sedikides, C. (2007). Temporal knowledge and autobiographical memory: An evolutionary perspective. In R. I. M. Dunbar \& L. Barrett (Eds.), Oxford handbook of evolutionary psychology (pp. 505-517). Oxford, UK: Oxford University Press.

Skowronski, J. J., Walker, W. R., \& Betz, A. L. (2003). Ordering our world: An examination of time in autobiographical memory. Memory, 11, 247-260.

Spreng, R. N., Mar, R. A., \& Kim, A. S. (2009). The common neural basis of autobiographical memory, prospection, navigation, theory of mind, and the default mode: A quantitative meta-analysis. Journal of Cognitive Neuroscience, 21, 489-510.

Spreng, R. N., Stevens, W. D., Chamberlain, J. P., Gilmore, A. W., \& Schacter, D. L. (2010). Default network activity, coupled with the frontoparietal control network, supports goal-directed cognition. Neuroimage, 53 , 303-317

St Jacques, P., Rubin, D. C., LaBar, K. S., \& Cabeza, R. (2008). The short and long of it: Neural correlates of temporal-order memory for autobiographical events. Journal of Cognitive Neuroscience, 20, 1327-1341.

Suddendorf, T. (2010). Episodic memory versus episodic foresight: Similarities and differences. Wiley Interdisciplinary Reviews. Cognitive Science, 1, 99-107.

Suddendorf, T., \& Corballis, M. C. (2007). The evolution of foresight: What is mental time travel and is it unique to humans? Behavioral and Brain Sciences, 30, 299-351. 
Summerfield, J. J., Hassabis, D., \& Maguire, E. A. (2010). Differential engagement of brain regions within a "core" network during scene construction. Neuropsychologia, 48, 1501-1509.

Szpunar, K. K. (2010). Episodic future thought: An emerging concept. Perspectives on Psychological Science, 5, 142-162.

Szpunar, K. K. (2011). On subjective time. Cortex, 47, 409-411.

Szpunar, K. K., Addis, D. R., McLelland, V. C., \& Schacter, D. L. (2013). Memories of the future: New insights into the adaptive value of episodic memory. Frontiers in Behavioral Neuroscience, 7, 47.

Szpunar, K. K., Watson, J. M., \& McDermott, K. B. (2007). Neural substrates of envisioning the future. Proceedings of the National Academy of Sciences, U.S.A., 104, 642-647.

Tulving, E. (2002). Episodic memory: From mind to brain. Annual Review of Psychology, 53, 1-25.
Viard, A., Desgranges, B., Eustache, F., \& Piolino, P. (2012). Factors affecting medial temporal lobe engagement for past and future episodic events: An ALE meta-analysis of neuroimaging studies. Brain and Cognition, 80, 111-125.

Vincent, J. L., Kahn, I., Snyder, A. Z., Raichle, M. E., \& Buckner, R. L. (2008). Evidence for a frontoparietal control system revealed by intrinsic functional connectivity. Journal of Neurophysiology, 100, 3328-3342.

Wheeler, M. A., Stuss, D. T., \& Tulving, E. (1997). Toward a theory of episodic memory: The frontal lobes and autonoetic consciousness. Psychological Bulletin, 121, 331-354.

Zhang, S., \& Li, C. S. (2012). Functional connectivity mapping of the human precuneus by resting state fMRI. Neuroimage, 59, 3548-3562. 